\title{
Analyse comparative structurale des diaphyses fémorales néandertaliennes BD 5 (MIS 5e) et CDV-Tour 1 (MIS 3) de La Chaise-de-Vouthon, Charente, France
}

Comparative structural analysis of the Neanderthal femoral shafts BD 5 (MIS 5e) and CDV-Tour 1 (MIS 3) from La Chaise-de-Vouthon, Charente, France

\section{Laurent Puymerail, Silvana Condemi et André Debénath}

\section{OpenEdition Journals}

Édition électronique

URL : http://journals.openedition.org/paleo/2676

DOI : $10.4000 /$ paleo.2676

ISSN : $2101-0420$

Éditeur

SAMRA

Édition imprimée

Date de publication : 15 décembre 2013

Pagination : 257-270

ISSN : $1145-3370$

Référence électronique

Laurent Puymerail, Silvana Condemi et André Debénath, « Analyse comparative structurale des diaphyses fémorales néandertaliennes BD 5 (MIS 5e) et CDV-Tour 1 (MIS 3) de La Chaise-de-Vouthon, Charente, France », PALEO [En ligne], 24 | 2013, mis en ligne le 17 avril 2014, consulté le 07 juillet 2020. URL : http://journals.openedition.org/paleo/2676 ; DOI : https://doi.org/10.4000/paleo.2676

Ce document a été généré automatiquement le 7 juillet 2020.

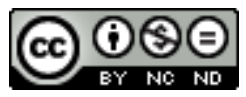

PALEO est mis à disposition selon les termes de la licence Creative Commons Attribution - Pas d'Utilisation Commerciale - Pas de Modification 4.0 International. 


\section{Analyse comparative structurale des diaphyses fémorales néandertaliennes BD 5 (MIS 5e) et CDV-Tour 1 (MIS 3) de La Chaise- de-Vouthon, Charente, France}

Comparative structural analysis of the Neanderthal femoral shafts BD 5 (MIS 5e) and CDV-Tour 1 (MIS 3) from La Chaise-de-Vouthon, Charente, France

Laurent Puymerail, Silvana Condemi et André Debénath

Nous remercions J.-F Tournepiche pour l'accès aux fossiles de La Chaise-de-Vouthon conservés au Musée d'Angoulême; A. Froment et P. Mennecier pour l'accès aux collections de fémurs fossiles et modernes du MNHN, Paris; A. Balzeau et P. Semal pour la disponibilité des registres tomographiques de La Ferrassie 2 et Spy II ; L. Bondioli pour l'accès aux collections du Musée "L. Pigorini », Rome ; à K. Chaumoitre et M. Panuel pour la disponibilité du registre tomographique (PACS) du service de radiologie de l'APHM, Marseille. L. Bondioli, E.A. Cabanis, J. Chiras, $G$. Trainaud et $P$. Vandermarcq ont assuré l'accès aux équipements $C T$ lors des acquisitions. Pour leur collaboration scientifique, nous remercions J. Braga, L. Fitton, $R$. Macchiarelli, F. Marchal, A. Mazurier, P. O'Higgins, B. Richmond, C.B. Ruff, P. Semal, J. Stock, E. Trinkaus, V. Volpato. Le Centre de Microtomographie de l'Université de Poitiers et la société NESPOS (www.nespos.org) ont fourni un appui technique essentiel.

\section{Introduction}

Par rapport à celle des humains modernes, la morphologie externe du fémur néandertalien adulte montre un contour périostéal subcirculaire au niveau de la région mi-diaphysaire, le développement variable d'un contrefort médial, une linea aspera relativement mince et l'absence de pilastre (Heim 1976, 1982 ; Trinkaus 1976 ; Trinkaus 
et Ruff 1999; Condemi 2001; Beauval et al. 2005). Même si associé à un degré de dimorphisme sexuel comparable à celui des populations humaines récentes (Trinkaus 1980 ; Walker et al. 2011), chez les Néandertaliens le fémur présente aussi une plus grande robustesse diaphysaire généralisée. Ce dernier aspect est compatible avec des charges biomécaniques plus élevées et une plus grande résistance aux contraintes orientées médio-latéralement, ainsi qu'une forte résistance axiale aux contraintes de torsion. Dans leur ensemble, ces caractéristiques représentent des adaptations également liées à la morphologie pelvienne et aux proportions corporelles propres aux Néandertaliens (Weaver 2003, 2009; Walker et al. 2011; Trinkaus et Ruff 2012). Les caractéristiques morphométriques de la partie externe de la diaphyse fémorale néandertalienne sont aujourd'hui bien documentées (cf. synthèse par Mussini et al. 2012). Toutefois, l'organisation de sa structure interne reste à explorer en termes fonctionnels, notamment pour l'os cortical. L'analyse de la variabilité topographique des épaisseurs corticales diaphysaires de fémurs néandertaliens de chronologie et de géographie diverses, peut apporter des informations biomécaniques pertinentes (Churchill 1998; Trinkaus et al. 1998; Trinkaus et Ruff 1999; Mussini et al. 2012 ; Puymerail et al. 2012a).

2 En association avec des modèles génétiques ayant suggéré l'existence de plusieurs sousgroupes au sein de la population néandertalienne à l'échelle de l'Europe continentale (Fabre et al. 2009 ; Hodgson, Bergey, Disotell 2010 ; Degioanni, Fabre, Condemi 2011 ; Dalen et al. 2012), des variations diachroniques dans la morphologie du squelette ont aussi été montrées à travers le Pléistocène supérieur (rev. dans Degioanni, Fabre, Condemi 2011; Di Vicenzo, Churchill, Manzi 2012; Stewart et Stringer 2012). Dans ce cadre, en plus de leur morphologie externe, nous avons extrait virtuellement, analysé et comparé les caractéristiques internes de deux diaphyses fémorales néandertaliennes - les spécimens BD 5 et CDV-Tour 1 - provenant du même site - La Chaise-de-Vouthon, en Charente - mais chronologiquement séparés d'environ 80000 ans (Condemi 2001; Puymerail et al. 2012a). Même si les deux spécimens sont incomplets (notamment, celui de la grotte de la Tour) et issus d'individus de sexe différent, à travers de nouveaux paramètres décrivant les variations fonctionnelles dans l'agencement de l'os cortical, cette étude préliminaire vise à identifier l'existence de possibles différences endostructurales entre diaphyses fémorales représentant des Néandertaliens ayant vécu du stade isotopique (MIS) 5 au MIS 3.

\section{Matériel et méthodes}

3 Le gisement de La Chaise-de-Vouthon est situé sur la rive gauche de la Tardoire, affluent de la Charente, sur une plate-forme en calcaire bathonien/bajocien caractérisée par une érosion intense qui a déterminé la formation d'une série d'abris sous roche et l'ouverture de plusieurs cavités karstiques. Les galeries se développent sur plusieurs dizaines de mètres et s'ouvrent sur l'extérieur par trois porches principaux, nommés abris Duport, Bourgeois-Delaunay et Suard (Debénath 1974, 1977, 2006).

4 La diaphyse fémorale BD 5 provient de l'abri Bourgeois-Delaunay (BD). Découvert dès 1850, cet abri est fouillé par les abbés Bourgeois et Delaunay en 1865, mais ce n'est que vers la fin des années 1930 que P. David entreprenait des fouilles systématiques qu'il poursuivra jusqu'en 1963. A la demande de J. Piveteau, A. Debénath a repris ensuite les 
recherches qui se sont échelonnées de 1967 à 1983 (Debénath 2006). Globalement, ce gisement couvre la période du Riss III jusqu'au Würm III (Debénath 1974, 1977, 2006 ; Schwarcz et Debénath 1979 ; Blackwell et al. 1983, 1992 ; Armand 1998). Des datations directes des spéléothèmes par ${ }^{230} \mathrm{Th} /{ }^{234} \mathrm{U}$ ont fourni des âges compris entre 127 et 116 ka (Couchoud 2006 ; Vieillevigne et al. 2008), qui correspondent au MIS 5e. Le spécimen BD 5, extrait en 1968 d'un bloc stalagmitique qui renfermait d'autres pièces crâniennes, représente un des 23 restes d'adultes et jeunes Néandertaliens, surtout des dents isolées, découverts au cours des fouilles les plus récentes et récoltés sur une surface d'environ $4 \mathrm{~m}^{2}$ (rev. dans Condemi 2001 ; Debénath 2006 ; Macchiarelli et al. 2006). Le spécimen représente une portion de fémur droit d'un jeune adulte, probablement de sexe féminin au regard de sa faible robustesse, conservée sur une longueur de 239,5 $\mathrm{mm}$, de la base du petit trochanter (trochanter minor) jusqu'au-dessous de la midiaphyse (Condemi 2001).

Découverte lors d'une exploration spéléologique, le spécimen CDV-Tour 1 provient de la grotte de la Tour, une cavité dont l'entrée est située à environ $130 \mathrm{~m}$ à l'ouest de l'Abri Suard. Le contexte de découverte est compatible avec un dépôt de tanière d'hyène et, sur base comparative à l'échelle régionale avec le registre d'autres sites objets de fouilles systématiques, l'assemblage des restes mammifères associés suggère une attribution chronologique au MIS 3 (Puymerail et al. 2012a). Ce fossile correspond à une portion diaphysaire fémorale gauche d'un individu adulte, vraisemblablement de sexe masculin vu sa forte robustesse, conservée sur une longueur de 204,7 $\mathrm{mm}$ et dont le contour total est préservé sur 95,8 mm (Puymerail et al. 2012a).

6 La morphologie externe de ces deux fossiles, qui sont conservés dans les collections du Musée d'Angoulême, est présentée en figure 1.

Figure 1 - Reconstruction virtuelle sur la base du registre microtomographique des spécimens BD 5 (à gauche) et CDV-Tour 1 (à droite) en vues antérieure, médiale, postérieure et latérale. Le niveau de la section mi-diaphysaire estimée est indiqué. Échelle $: 2 \mathrm{~cm}$.

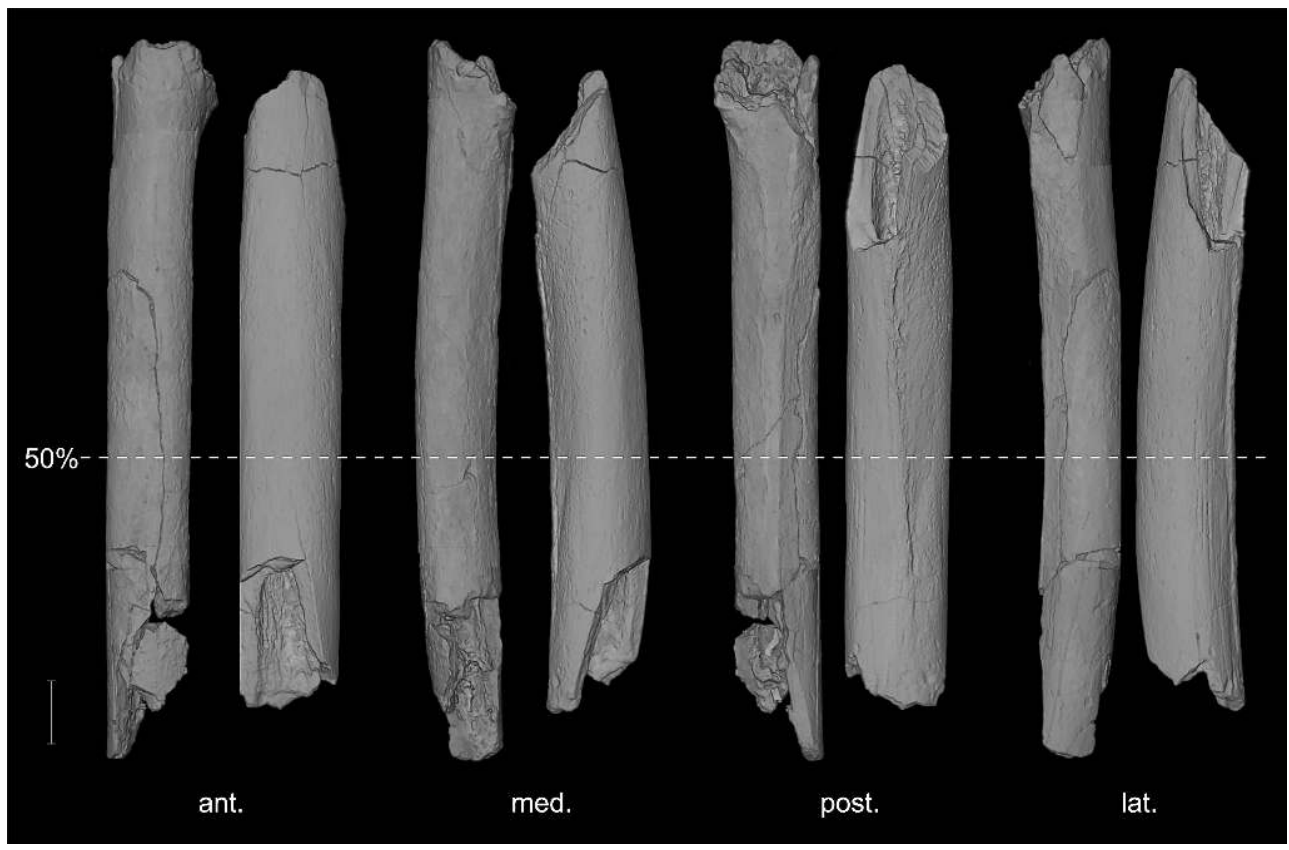

BD 5 et CDV-Tour 1 ont été microtomographiés $(\mu \mathrm{CT})$ en 2008 au Centre de Microtomographie de l'Université de Poitiers. Pour détailler les spécimens dans leur 
intégralité, les acquisitions se sont déroulées en trois fois pour BD 5 et en deux fois pour CDV-Tour 1. Les acquisitions ont été réalisées avec un équipement X8050-16 multi-échelle Viscom A G (caméra $1004 \times 1004$ de 12 bit avec une taille de pixel de 147 $\mu \mathrm{m}$ ), selon 1200 projections (tous les $0,30^{\circ}$ ) et 32 intégrations/projection. Pour BD 5, une énergie de $100 \mathrm{kV}$ et une intensité de $580 \mu \mathrm{A}$ ont été utilisées, alors que les mêmes paramètres correspondent à $120 \mathrm{kV}$ et $560 \mu \mathrm{A}$ dans le cas de CDV-Tour 1. Le volume final d'images a été reconstruit par l'intermédiaire du logiciel DigiCT v.1.15 (DIGISENS) à une taille de voxel isométrique de $86,3 \mu \mu \mathrm{m}$ pour BD 5 et de $107,8 \mu \mathrm{m}$ pour CDV-Tour 1.

8 La bonne conservation des contours périostéal et endostéal des diaphyses, associée dans les deux cas à l'absence de remplissage sédimentaire de la cavité médullaire, a permis l'application de la méthode de seuillage global (Spoor, Zonneveld, Macho 1993), en mesurant la moyenne de l'interface os/air sur une vingtaine de coupes du lot d'images (Fajardo, Ryan, Kapelman 2002 ; Coleman et Colbert 2007). La segmentation semi-automatique avec corrections manuelles a été effectuée à l'aide des logiciels Avizo v.7.0.0 (Visualization Sciences Group Inc.) et ImageJ v.1.46a (Rasband 2010). Le résultat de la segmentation est un maillage triangulaire des surfaces périostéale et endostéale composées de coordonnées 3D reliées par des lignes qui génèrent des faces triangulaires obtenues après lissage. Le lissage (marching cube) a été effectué à l'aide du logiciel Avizo v. 7.0.0 (option unconstrained smoothing) sans que cela n'influence la sensibilité des analyses et des résultats.

9 Pour orienter anatomiquement les deux spécimens, nous nous sommes basés sur la position de la linea aspera, appréciable dans les deux spécimens. Nous avons ensuite localisé la section à environ $50 \%$ en se basant sur la morphologie et le trajet de cette ligne examinée en projection médiale et latérale. Par rapport aux humains modernes, la localisation précise de la mi-diaphyse est généralement moins nette dans les fémurs néandertaliens à contour subcirculaire et dépourvus de pilastre. En revanche, cette région diaphysaire ne présente que peu de changements morpho-structuraux sur plusieurs centimètres, ce qui rend marginales les erreurs de sa localisation et les analyses associées (Beauval et al. 2005 ; Sládek et al. 2010 ; Puymerail et al. 2012a, b ; Trinkaus et Ruff 2012).

10 Pour estimer la longueur biomécanique de BD 5, nous avons déterminé sur le registre $\mu \mathrm{CT}$ la position de la section se trouvant à $1 \mathrm{~cm}$ à la base du petit trochanter, qui par définition correspond à $80 \%$ de la longueur biomécanique (Ruff et al. 1999). Ensuite, en prenant aussi en compte la position de la mi-diaphyse, nous avons pu déterminer la section distale, dont tout le contour est conservé à environ $37 \%$ de la longueur biomécanique. Le protocole suivi pour mesurer le spécimen CDV-Tour 1, qui a permis de localiser la section proximale à $65 \%$ et celle distale à ca. $42 \%$ de la longueur biomécanique, a été récemment décrit en détail (Puymerail et al. 2012a).

11 A l'aide d'une routine développée en langage R v.2.11.1 (R Development Core Team 2011), les paramètres de géométrie de section suivants ont été mesurés/calculés chaque $1 \%$ le long des ces deux diaphyses néandertaliennes : aire totale (TA, en $\mathrm{mm} 2)$; aire corticale (CA, en mm2) ; pourcentage d'aire corticale (\% CA) ; second moment de l'aire selon les axes médio-latéral (m-l) et antéro-postérieur (a-p) (Ix, Iy, en $\mathrm{mm} 4)$; second moment polaire d'aire (J, en $\mathrm{mm} 4$ ) ; second moment d'aire maximal et minimal (Imax, Imin, en mm4) (pour tous détails méthodologiques, voir Ruff 2008). 
12 Pour replacer les deux spécimens de La Chaise dans un contexte de variations chronogéographiques, en plus des diamètres diaphysaires a-p et m-1 (M6, M7, M9, M10 ; Martin et Saller 1956-1962) et des indices pilastrique (à $50 \%$ ) et platymérique (à $80 \%$ ), nous avons comparé leurs propriétés géométriques de section mesurées à $50 \%$ et à $65 \%$ (pour les deux spécimens) et à $80 \%$ (seulement pour BD5) au registre fossile disponible pour 22 fémurs d'adultes néandertaliens de l'Europe centre-occidentale répartis sans distinction de sexe selon leur chronologie du MIS 5 au MIS 3 (tabl. 1). Ces données comparatives proviennent en large mesure du travail de synthèse récemment publié par Trinkaus et Ruff (2012) et de l'étude du spécimen français des Pradelles (Mussini et al. 2012). Les humains modernes sont ici représentés par un large échantillon de 102 fémurs mesurés à partir d'un registre tomographique médical (CT) réalisé à une résolution variant entre 350 et $976 \mu \mathrm{m}$ (cf. Puymerail 2011; Puymerail et al. 2012c). Cet échantillon est composé d'adultes des deux sexes issus des collections ostéologiques françaises du XIXe, du site archéologique d'époque romaine de Velia (Italie) et du system PACS de l'Assistance Publique des Hôpitaux de Marseille.

Dans une perspective fonctionnelle, des avancées méthodologiques dans le domaine de l'imagerie 3D permettent désormais le déroulement virtuel de la surface diaphysaire externe et sa projection sous forme de cartographie morphométrique représentant les variations topographiques des épaisseurs du tissu cortical à travers et le long de la portion osseuse déroulée (Bondioli et al. 2010; Puymerail et al. 2012a, b). D’abord, l'épaisseur est définie pour chaque point de la surface périostéale comme la distance par rapport au point le plus proche sur la surface interne (Mazurier, Nakatsukasa, Macchiarelli 2010 ; Bayle et al. 2011; Volpato et al. 2011, 2012, 2013; Puymerail et al. 2012a, b). La cartographie des épaisseurs est ensuite projetée sur la surface d'un cylindre, dont la largeur correspond au diamètre maximal de la diaphyse, qui est virtuellement déroulée à partir d'un axe passant par le centre de la face antérieure de l'os (Bondioli et al. 2010). Dans une perspective comparative, dans cette étude nous avons considéré les cartographies morphométriques des fémurs néandertaliens La Ferrassie 2 (droit) et Spy II (droit) (dans Puymerail et al. 2012a), respectivement attribués aux MIS 5 (Jaubert et al. 2010 ; mais voir aussi Blackwell et al. 2007 ; Bertran et al. 2008) et MIS 3 (Semal et al. 2009). Pour générer une cartographie unique et consensuelle de notre échantillon de 102 humains modernes, nous avons utilisé des techniques de géostatistique de krigeage et de GAM ("generalized additive model »). Pour ce faire, à travers l'utilisation des librairies mgcv (Wood 2006), spatstat (Baddeley 2008) et gstat (Pebesma 2004), nous avons utilisé le modèle de répartition des épaisseurs relatives obtenu grâce à une routine développée en langage Scilab v.4.1.2 (Consortium Scilab) et en R v.2.11.1 (R Development Core Team 2011).

14 Comme l'ont montré des études similaires sur la modélisation 3D des os longs en utilisant différents systèmes d'imagerie (Bondioli et al. 2010; Mazurier et al. 2010 ; Bayle et al. 2011 ; Volpato et al. 2011, 2012, 2013 ; Puymerail et al. 2012a, b), un certain nombre de comparaisons inter et intra-observateurs ont révélé des différences inférieures ou égales à $5 \%$. 


\section{Résultats et Discussions}

\section{Morphologie externe}

15 La diaphyse fémorale BD 5 est assez gracile et sans rétrécissement mi-diaphysaire et présente une convexité médiale peu prononcée associée à une antérieure plus nette. En association à l'absence de pilastre, le contour périostéal est subcirculaire. L'extrémité proximale est incomplète mais la présence d'un petit trochanter nettement individualisé indique l'insertion puissante du muscle psoas iliaque. La linea aspera est également bien marquée et présente dans sa portion supérieure un relief épais et mousse mais qui n'est pas partagé par une gouttière (Condemi 2001). Malgré des différences évidentes de taille entre les deux fossiles (qui atteint environ $52 \%$ pour l'aire totale de la section à mi-diaphyse et $46 \%$ pour celle à $65 \%$ ), ces caractéristiques morphologiques se retrouvent représentées également chez CDV-Tour 1. En effet, ce spécimen du MIS 3 présente de légères courbures antérieure et médiale. Le contour de sa région mi-diaphysaire varie entre une légère extension selon l'axe $\mathrm{m}-\mathrm{l}$ et une section subcirculaire. La linea aspera montre deux lèvres fusionnant pour former une crête relativement lisse au niveau proximal (Puymerail et al. 2012a).

Les valeurs comparatives des diamètres $a-p$ et $m-1$ sont présentées dans le tableau 2 , alors que la variation des indices pilastriques et platymériques est illustrée en figure 2 . La décomposition de ces deux indices en rapports de diamètres nous permet de mettre en évidence les effets d'allométrie. Ainsi, avec des valeurs de l'indice pilastrique respectivement de 96,5 et 93,7, BD 5 et CDV-Tour 1 s'inscrivent en limites supérieure et inférieure du nuage néandertalien, qui est bien identifié par rapport à celui des humains modernes (fig. 2a). Ètant caractérisé par des valeurs relativement faibles des diamètres a-p (M6) et m-l (M7), BD 5 se rapproche des individus considérés de sexe féminin, comme Palomas 96 (Walker et al. 2011) et La Ferrassie 2 (Heim 1976, 1982) ; au contraire, à cause du très fort diamètre $\mathrm{m}-\mathrm{l}, \mathrm{CDV}$-Tour 1 se rapproche des individus Saint-Césaire 1 (Trinkaus et al. 1998) et La Ferrassie 1 (Heim 1976, 1982). En ce qui concerne les différences entre les échantillons au regard de leur distribution chronologique, on remarque que, par rapport à l'ensemble des 10 spécimens qui représente le MIS 3, les fémurs attribués au MIS 5 montrent en proportion d'assez faibles valeurs du diamètre $a-p$, associées à des valeurs assez élevées du diamètre $m-1$. Dans cette étude, le MIS 4 n'est représenté que par deux spécimens qui apparaissent très proches pour leurs valeurs élevées du diamètre a-p (fig. 2a). En ce qui concerne la section sub-trochantérienne ( $80 \%$ ), nous ne pouvons que comparer BD 5 , car cette portion n'est pas conservée chez CDV-Tour 1. Dans ce cas encore, les valeurs de diamètres a-p et $\mathrm{m}-\mathrm{l}$ placent le spécimen de l'abri Bourgeois-Delaunay parmi les fémurs néandertaliens les plus graciles, comme Krapina 214 et Tabun 1 (Trinkaus et Ruff 2012). A ce niveau diaphysaire, on ne remarque pas de différences entre les sous échantillons du MIS 5 et 3, alors que les représentants du MIS 4 présentent des valeurs du diamètre a-p parmi les plus élevées en absolu.

Cette variabilité biométrique est bien synthétisée par les variations des indices pilastrique et platymérique présentés en figure $2 \mathrm{~b}$. 
Figure 2 - Mesures comparatives des diamètres a-p vs. m-l à $50 \%$ et à $80 \%$ (a) et valeurs des indices pilastrique et platymérique (b) pour BD 5 (triangle noir), CDV-Tour 1 (étoile noire), trois échantillons néandertaliens du MIS 5, 4, 3 et un échantillon d'humains modernes (EH) (cf. tableau 2). Les ellipses rassemblent $95 \%$ de la variabilité.
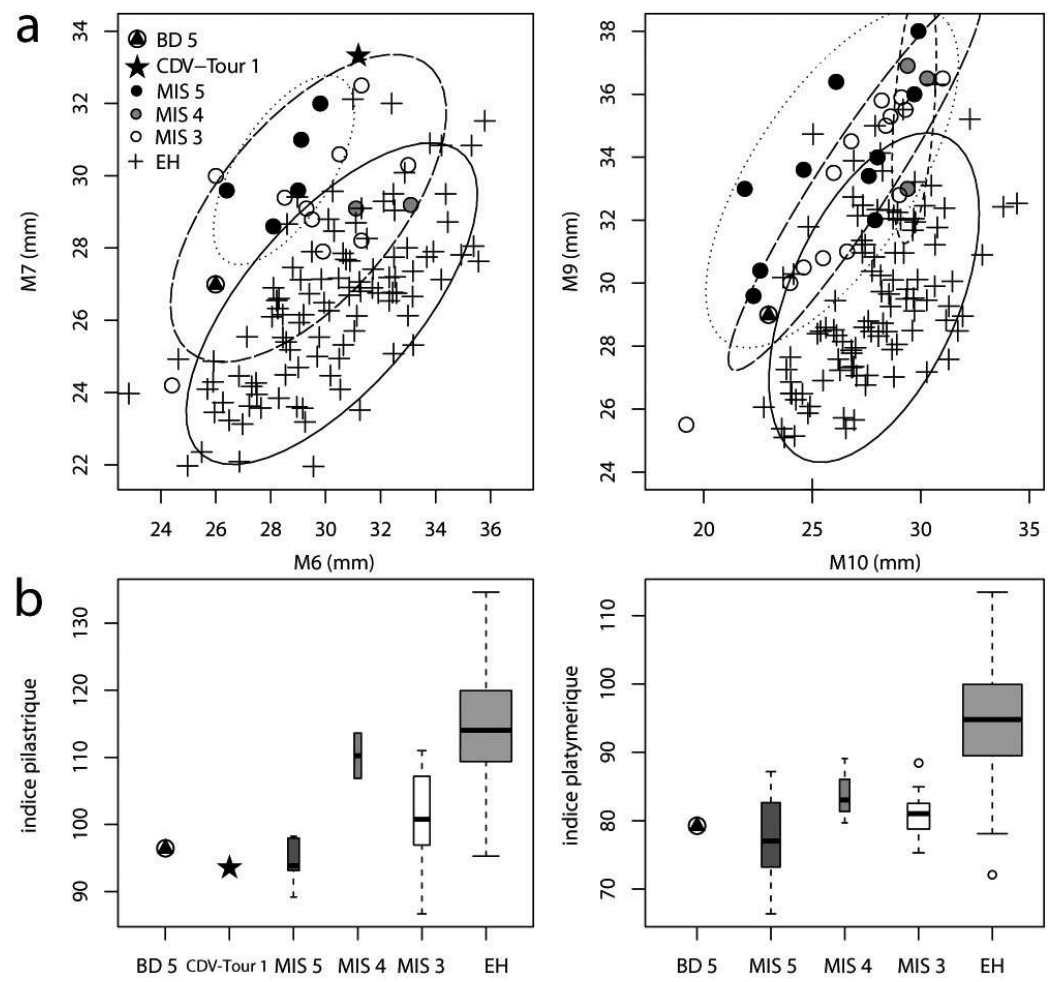

Tableau 1 - Distribution chronologique (MIS 5-3) des spécimens néandertaliens utilisés dans l'analyse comparative des diamètres a-p et $\mathrm{m}$-I mesurés à mi-diaphyse $(*)$ et à $80 \%(\#)$ de la longueur biomécanique ; des paramètres de géométrie de section mesurés à $50 \%$ (!), à $65 \%$ (§) et à $80 \%(\mathrm{a})$ de la diaphyse fémorale. Données extraites de Trinkaus et Ruff $(2012$, tableaux A4, A5, A6 et A13; voir aussi Mussini et al. 2012).

\begin{tabular}{|c|c|}
\hline MIS & spécimens \\
\hline 5 & $\begin{array}{l}\text { Ferrassie } 1 * \# ! \S \propto, \text { Ferrassie } 2 * \# ! \S \propto, \text { Krapina 213\#, Krapina 214\#, Krapina 257.32\#ם, } \\
\text { Krapina 257.33\#ם, Stadelhole } 1 * \#\end{array}$ \\
\hline 4 & Les Pradelles*!, Chapelle-aux-Saints $1 * \# !$ \\
\hline 3 & 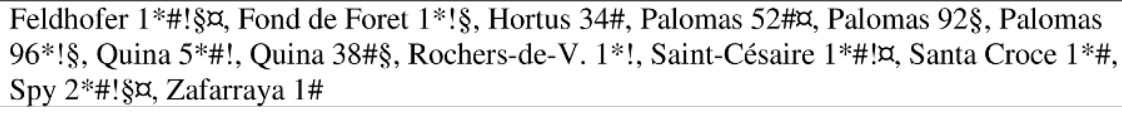 \\
\hline
\end{tabular}

\section{Morphologie interne}

Les valeurs des propriétés géométriques de sections des deux spécimens de La Chaisede-Vouthon et des échantillons comparatifs sont détaillées en tableau 2. A ce propos on note qu'aucune valeur n'est disponible pour l'échantillon néandertalien du MIS4 à $65 \%$ de la longueur biomécanique fémorale. Pour les spécimens néandertaliens BD 5, CDVTour 1, La Ferrassie 2 et Spy II et pour l'échantillon humain moderne utilisé dans cette étude (pour lesquels nous possédons les données individuelles 3D du registre [micro]tomographique), la figure 3 présente les variations des paramètres \% CA et ratio Ix/Iy mesurés tous les pourcent le long de la diaphyse. Pour \%CA, l'ensemble des spécimens fossiles et modernes présentent une diaphyse plus robuste dans sa portion proximale (notamment, dans la région 65-70\%) par rapport à la moitié distale du fémur (fig. 3a). Dans ce contexte, CDV-Tour 1 s'inscrit dans la variabilité moderne, alors que 
BD 5 présente des valeurs de robustesse supérieures sur toute la portion conservée, sauf pour la partie la plus proximale. On remarque également que ce dernier fémur présente un profil de variation proche du spécimen La Ferrassie 2, également du MIS 5 et considéré de sexe féminin, et qu'une substantielle homogénéité des propriétés biomécaniques existe entre CDV-Tour 1 et Spy II, tous deux issus du MIS 3.

Figure 3 - Pourcentage d'aire corticale (a) et rapport Ix/ly (b) mesurés à intervalles réguliers de $1 \%$ le long de la diaphyse fémorale dans la portion comprise entre $20 \%$ et $80 \%$ de la longueur biomécanique de BD 5, CDV-Tour 1, La Ferrassie 2, Spy II et dans un échantillon d'humains modernes (EH).

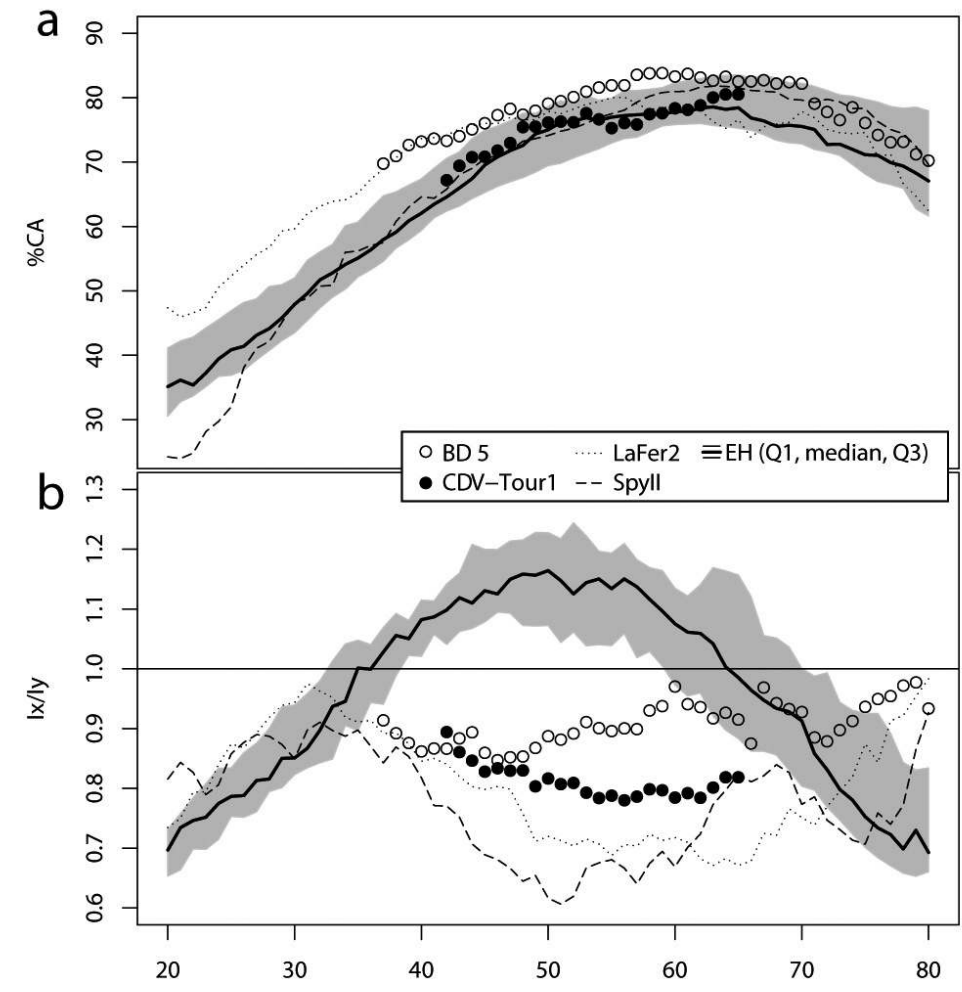

Les valeurs du ratio Ix/Iy, qui traduit la résistance à la flexion par rapport aux axes antéro-postérieur et medio-latéral (a-p / $\mathrm{m}-\mathrm{l})$, sont présentées en figure $3 \mathrm{~b}$. Pour ce paramètre, les valeurs supérieures à l'unité indiquent une résistance majeure selon l'axe a-p, alors que celles inférieures à 1,0 indiquent une résistance majeure selon l'axe $\mathrm{m}-\mathrm{l}$. Dans ce cas, la signature endostructurale néandertalienne est clairement distincte par rapport à celle des humains modernes. En effet, la diaphyse fémorale des humains modernes est caractérisée par une forte résistance aux contraintes orientées selon l'axe $\mathrm{m}-\mathrm{l}$ au niveau des sections les plus distales et les plus proximales, alors que la présence caractéristique d'un pilastre entraîne une plus grande résistance aux contraintes selon l'axe a-p au niveau mi-diaphysaire. Par rapport à ce schéma structural, sans différence apparente d'ordre chronologique, les quatre Néandertaliens considérés dans cette analyse présentent tous des valeurs du ratio Ix/Iy inférieures à l'unité pour la portion 35-65 (fig. 3b).

19 Nous avons également étudié la distribution comparative du second moment de l'aire selon l'axe a-p (Ix) et selon l'axe m-l (Iy) à $50 \%, 65 \%$ et $80 \%$ de la longueur biomécanique estimée (tabl. 2 et fig. 4). Comme nous l'avions décrit pour les diamètres a-p et m-l à mi-diaphyse (fig. 2a), à 50 \% et $65 \%$ BD 5 tombe avec Palomas 96 (Walker et 
al. 2011) à la limite inférieure de la distribution néandertalienne, alors que CDV-Tour 1 se retrouve lui parmi les spécimens les plus robustes, dont La Ferrassie 1 (Heim 1976, 1982) et Fond-de-Forêt (Trinkaus et Ruff 1989). Au niveau de ces deux sections, les Néandertaliens attribués au MIS 5 présentent des valeurs de Iy relativement élevées. A 80 \% (donnée non disponible pour CDV-Tour 1), le fémur de l'abri Bourgeois-Delaunay présente à nouveau des caractéristiques indiquant une faible robustesse par rapport à la moyenne néandertalienne et se rapproche encore de Krapina 214 (Trinkaus et Ruff 2012). Dans ce cadre, comme il a été déjà indiqué pour d'autres spécimens néandertaliens (Trinkaus 1980), la gracilité de BD 5 et la robustesse biomécanique de CDV-Tour 1 relèvent du dimorphisme sexuel. A $50 \%$ comme à $65 \%$ de la longueur biomécanique, notre échantillon néandertalien montre des caractéristiques bien identifiables par rapport aux propriétés structurales caractérisant l'échantillon moderne. En effet, chez les Néandertaliens les contraintes selon l'axe m-l sont systématiquement supérieures à celles selon l'axe a-p. Toutefois, ce patron exprime une plus forte variabilité au niveau de la section proximale, notamment par rapport aux fortes contraintes m-l (fig. 4a), ce qui vraisemblablement reflète l'influence directe de la morphologie pelvienne propre aux Néandertaliens (Ponce de León et al. 2008 ; Weaver et Hublin 2009 ; Meyer et al. 2011 ; Trinkaus 2011 ; Trinkaus et Ruff 2012). En ce qui concerne le pourcentage d'aire corticale ( $\% C A)$, à $50 \%(79,1$ vs. 76,2$)$ et à $65 \%(83,2$ vs. 80,5 ) BD 5 est légèrement plus robuste que CDV-Tour 1 (fig. $4 \mathrm{~b}$ ). En revanche, à $80 \%$ les valeurs de \%CA de BD 5 ne permettent pas de distinguer ce spécimen des échantillons néandertaliens et moderne.

Tableau 2 - Mesures comparatives des diamètres diaphysaires a-p et $\mathrm{m}-\mathrm{l}$ et valeurs brutes des neuf paramètres de géométrie de section (voir le texte pour la signification des abréviations) mesurés à $80 \%, 65 \%$ et $50 \%$ de la longueur biomécanique dans les deux spécimens BD 5 et CDV-Tour 1 de La Chaise-de-Vouthon et dans trois échantillons néandertaliens du MIS 5, 4, 3. Les humains modernes sont représentés par l'échantillon EH. Pour chaque échantillon sont présenté la valeur moyenne et l'écart type (en italique). Le nombre de cas considérés (n) est systématiquement spécifié pour les diamètres diaphysaires $(\varnothing)$ et les propriétés géométrique de section (PGS). Données extraites de Trinkaus et Ruff (2012, tableaux A4, A5, A6 et A13; voir aussi Mussini et al. 2012).

\begin{tabular}{|c|c|c|c|c|c|c|c|c|c|c|c|c|}
\hline section & spéc./échant. & a-p & $\mathrm{m}-\mathrm{l}$ & TA & $\mathrm{CA}$ & $\% \mathrm{CA}$ & Ix & Iy & $\mathrm{Ix} / \mathrm{Iy}$ & $\mathbf{J}$ & Imax & Imin \\
\hline \multirow[t]{9}{*}{$80 \%$} & BD 5 & 23 & 29 & 572 & 401 & 70,2 & 23553 & 25230 & 0,93 & 48784 & 29441 & 19352 \\
\hline & \multirow{2}{*}{$\begin{array}{c}\text { MIS 5 } \\
\text { nØ:10; nPGS: } \\
5\end{array}$} & 25,9 & 33,3 & 741 & 561 & 76,7 & 35721 & 51521 & 0,69 & 87241 & 54312 & 32930 \\
\hline & & 2,9 & 2,8 & 156 & 107 & 10,3 & 14021 & 18601 & 0,16 & 31530 & 19321 & 12743 \\
\hline & \multirow{2}{*}{$\begin{array}{c}\text { MIS } 4 \\
\text { nø:3; nPGS:1 }\end{array}$} & 29,7 & 35,4 & 797 & 634 & 79,5 & 46481 & 51455 & 0,90 & 97936 & 51467 & 46469 \\
\hline & & 0,5 & 2,1 & $N A$ & $N A$ & $N A$ & $N A$ & $N A$ & $N A$ & $N A$ & $N A$ & $N A$ \\
\hline & \multirow{2}{*}{\begin{tabular}{c|c} 
MIS 3 \\
$\mathrm{n} \emptyset: 14 ; \mathrm{nPGS}: 3$ \\
\end{tabular}} & 26,9 & 33,2 & 777 & 565 & 73,9 & 39773 & 52815 & 0,75 & 92587 & 55076 & 37511 \\
\hline & & 2,9 & 3,1 & 142 & 82 & 10,1 & 13070 & 15703 & 0,15 & 27636 & 15930 & 12826 \\
\hline & \multirow{2}{*}{$\begin{array}{c}\text { EH } \\
\text { nØ:102; } \\
\text { nPGS: } 102 \\
\end{array}$} & 27,9 & 29,5 & 639 & 432 & 67,8 & 28385 & 32876 & 0,87 & 61261 & 34751 & 26512 \\
\hline & & 2,5 & 2,7 & 99 & 67 & 7,1 & 8809 & 9936 & 0,14 & 17853 & 10254 & 8067 \\
\hline \multirow[t]{8}{*}{$65 \%$} & BD 5 & 25,8 & 27,1 & 510 & 425 & 83,2 & 20096 & 21693 & 0,91 & 41790 & 23694 & 18086 \\
\hline & \multirow{3}{*}{$\begin{array}{c}\text { CDV-Tour } 1 \\
\text { MIS } 5 \\
\text { nPGS:7 }\end{array}$} & 31,6 & 33,7 & 745 & 600 & 80,5 & 39294 & 48004 & 0,81 & 87298 & 51387 & 35883 \\
\hline & & & & 596 & 499 & 84,1 & 25258 & 32768 & 0,82 & 58026 & 34316 & 23710 \\
\hline & & & & 120 & 94 & 6,5 & 8776 & 13276 & 0,22 & 21152 & 12855 & 8942 \\
\hline & \multirow{2}{*}{$\begin{array}{c}\text { MIS } 3 \\
\text { nPGS:6 }\end{array}$} & & & 603 & 504 & 84,0 & 26193 & 32995 & 0,84 & 59188 & 34982 & 24206 \\
\hline & & & & 111 & 87 & 6,0 & 8359 & 12258 & 0,21 & 19668 & 11851 & 8519 \\
\hline & \multirow{2}{*}{$\begin{array}{c}\text { EH } \\
\text { nø:102; } \\
\text { nPGS:102 }\end{array}$} & 29,3 & 26,4 & 582 & 458 & 78,9 & 30002 & 24602 & 1,23 & 54605 & 31191 & 23409 \\
\hline & & 2,6 & 2,3 & 91 & 73 & 5,8 & 9828 & 7830 & 0,22 & 16812 & 9935 & 7311 \\
\hline \multirow[t]{10}{*}{$50 \%$} & BD 5 & 26 & 27 & 488 & 385 & 79,1 & 17456 & 19676 & 0,88 & 37133 & 19714 & 17395 \\
\hline & CDV-Tour 1 & 31,2 & 33,3 & 744 & 566 & 76,2 & 37844 & 46344 & 0,81 & 84188 & 46327 & 37839 \\
\hline & MIS 5 & 28,5 & 30,1 & 669 & 522 & 77,9 & 31426 & 38085 & 0,82 & 69511 & 41419 & 28092 \\
\hline & nØ:5; nPGS:4 & 1,3 & 1,3 & 65 & 60 & 1,4 & 6673 & 7553 & 0,06 & 13882 & 8888 & 5469 \\
\hline & \multirow{2}{*}{$\begin{array}{c}\text { MIS } 4 \\
\text { nø:2; nPGS:2 }\end{array}$} & 32,1 & 29,1 & 713 & 591 & 82,9 & 36172 & 42350 & 0,85 & 78023 & 44379 & 34143 \\
\hline & & $N A$ & $N A$ & $N A$ & $N A$ & $N A$ & $N A$ & $N A$ & $N A$ & $N A$ & $N A$ & $N A$ \\
\hline & \multirow{2}{*}{\begin{tabular}{c|} 
MIS 3 \\
nØ:10; nPGS:7
\end{tabular}} & 29,4 & 29,1 & 645 & 513 & 79,0 & 32134 & 33585 & 0,95 & 65720 & 37883 & 27837 \\
\hline & & 2,5 & 2,7 & 89 & 90 & 5,5 & 9032 & 8466 & 0,14 & 16625 & 107550 & 6448 \\
\hline & \multirow{2}{*}{$\begin{array}{c}\text { EH } \\
\text { nØ:102; } \\
\text { nPGS: } 102\end{array}$} & 30,2 & 26,4 & 577 & 427 & 74,2 & 30618 & 22916 & 1,34 & 53535 & 31216 & 22319 \\
\hline & & 2,7 & 2,3 & 90 & 70 & 5,3 & 9960 & 7093 & 0,21 & 16476 & 9935 & 6954 \\
\hline
\end{tabular}


Figure 4 - Mesures comparatives du second moment de l'aire Ix vs. ly (a) et du pourcentage d'aire corticale (b) à $50 \%$, à $65 \%$ et à $80 \%$ de la longueur biomécanique pour BD 5 (triangle noir), CDVTour 1 (étoile noire) et trois échantillons néandertaliens du MIS 5, 4, 3 et un échantillon d'humains modernes (EH) (cf. tableau 2). Les ellipses rassemblent $95 \%$ de la variabilité.
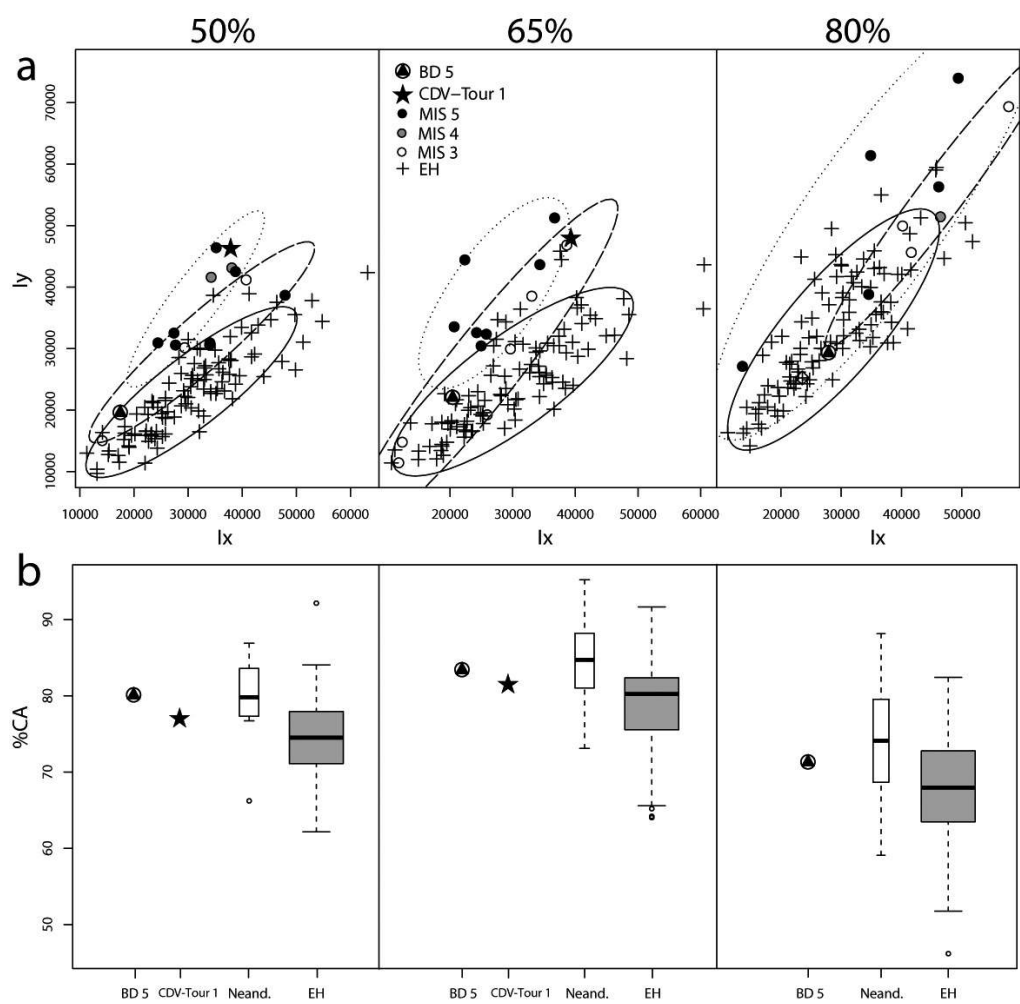

Tout comme pour d'autres fémurs néandertaliens, les diaphyses BD 5 et CDV-Tour 1 sont caractérisées par une résistance à des contraintes relativement importantes selon l'axe $\mathrm{m}-\mathrm{l}$, ce qui se traduit par la présence d'un renforcement médial au niveau de la portion mi-diaphysaire évident dans les deux spécimens (fig. 5). A partir du registre (micro)tomographique, le déroulement virtuel de la diaphyse et les cartographies des variations topographiques de l'os cortical permet une caractérisation morphofonctionnelle plus fine de cette particularité néandertalienne (fig. 6). Ici, nous avons comparé la cartographie morphométrique des fémurs BD 5 (portion $~ 37-80 \%$ ) et CDVTour 1 (portion 42-65\%) à celles obtenues pour les spécimens La Ferrassie 2 et Spy II (Puymerail et al. 2012a; Volpato et al. 2013), qui ont été générées pour la portion comprise entre $20 \%$ et $80 \%$ de la longueur biomécanique. Dans notre analyse, les humains modernes sont représentés par une unique cartographie consensuelle qui résume l'ensemble des informations endostructurales de notre large référentiel de 102 fémurs adultes. Cette approche méthodologique, où chaque cartographie morphométrique représente les variations relatives dans la distribution topographique du tissu cortical, décrit les schémas de distribution plutôt que les épaisseurs ellesmêmes (Puymerail et al. 2012a). Dans ce contexte comparatif (fig. 6), BD 5 présente un renforcement structural oblique situé le long de la face médiale, associé à un fin renfort le long de la face postérieure correspondant au relief de la linea aspera. Les épaisseurs les plus fortes sont mesurées au niveau proximal de la face latérale et correspondent à l'insertion des muscles fessiers (Mariotti et Belcastro 2011 ; Puymerail et al. 2012a). La portion conservée de CDV-Tour 1 est bien moins étendue. Néanmoins, on y remarque également le renfort médial oblique présent sur BD 5, associé aussi à un renfort 
postérieur. Ces particularités endostructurales, qui caractérisent bien le modèle néandertalien par rapport aux humains modernes, sont partagées avec les autres spécimens fossiles utilisés dans notre analyse, sans différence visible par rapport à leur contexte chronologique ou au dimorphisme sexuel. Alors qu'un renforcement médiolatéral diaphysaire a été révélé par la géométrie de section, la cartographie morphométrique permet de préciser la contribution surtout médiale de ce contrefort. Comme a déjà été évoqué, BD 5 représenterait une jeune femme adulte (Condemi 2001), alors que CDV-Tour 1 un individu robuste de sexe masculin (Puymerail et al. 2012a). Néanmoins, à la différence des mesures diaphysaires de section (diamètres, aires), l'analyse de la distribution topographique du tissu cortical ne met pas en évidence de différences attribuables au dimorphisme sexuel.

Figure 5 - Reconstruction virtuelle des spécimens BD 5 (à gauche) et CDV-Tour 1 (à droite) en vues antérieure, médiale, postérieure et latérale. Les variations d'épaisseur de l'os cortical de la portion comprise entre environ $37 \%$ et $80 \%$ de la longueur biomécanique pour BD 5 et entre environ $42 \%$ et $65 \%$ pour CDV-Tour 1 sont représentées selon une échelle chromatique (en bleu les valeurs les plus fines, en rouge les plus épaisses). Le niveau de la section mi-diaphysaire estimée est indiqué. Échelle : $2 \mathrm{~cm}$.

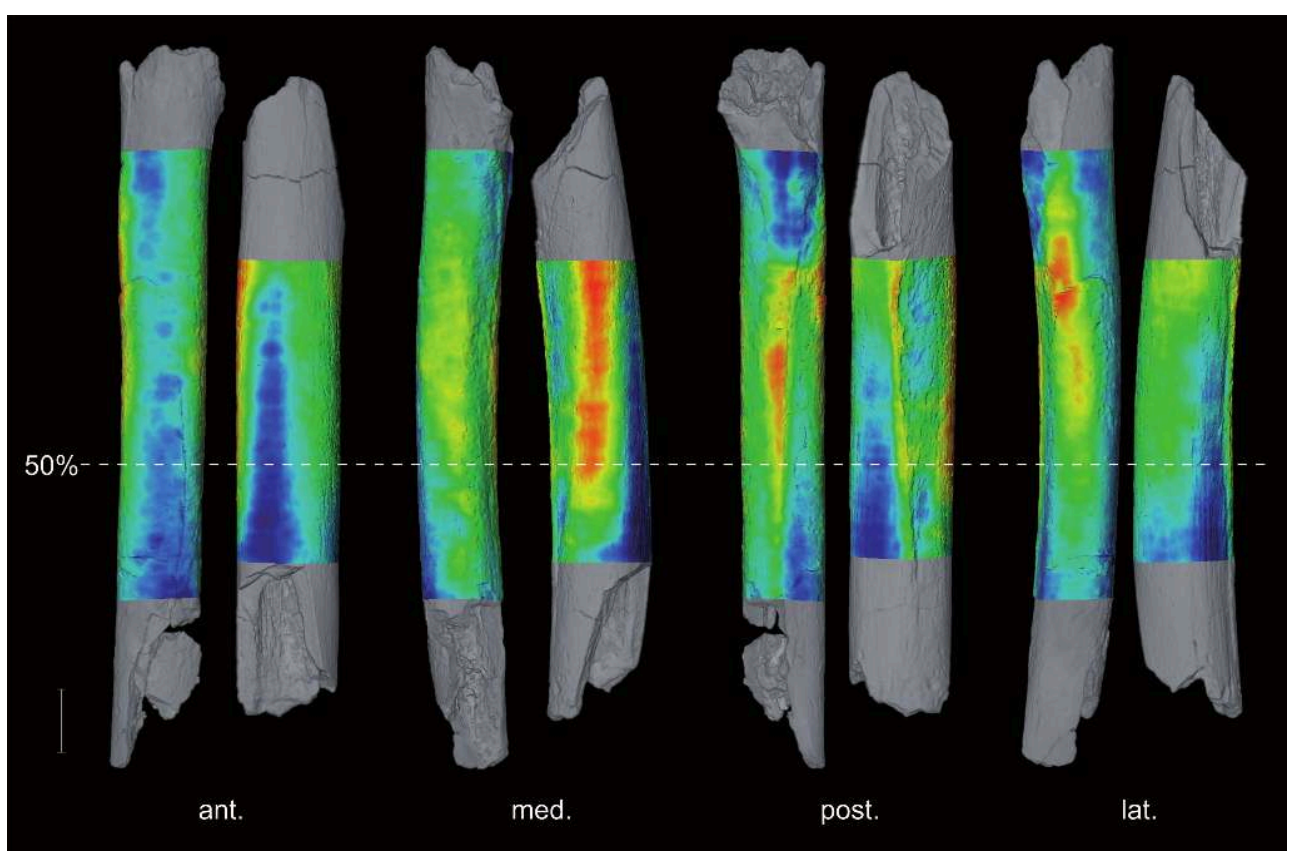


Figure 6 - Cartographies des variations topographiques standardisées de l'épaisseur de l'os cortical de BD 5 (portion 37-80 \%) et CDV-Tour 1 (portion 42-65\%) comparée à celles des spécimens néandertaliens La Ferrassie 2 (droit) et Spy II (droit) et au consensus de l'échantillon d'humains modernes $(E H)$. Chaque fémur a été virtuellement découpé verticalement le long de la face antérieure puis déroulé. Indépendamment de leur latéralité, tous les spécimens sont représentés comme gauche ; le point de vue est systématiquement de l'intérieur vers l'extérieur (côté médial à gauche). Chaque cartographie est représentée à l'aide d'une grille de 100 colonnes (x) par 200 lignes (y). ant. : antérieur ; lat. : latéral ; med. : médial ; post. : postérieur. Épaisseur représentée selon une échelle chromatique croissante du bleu foncé (0) au rouge (1).

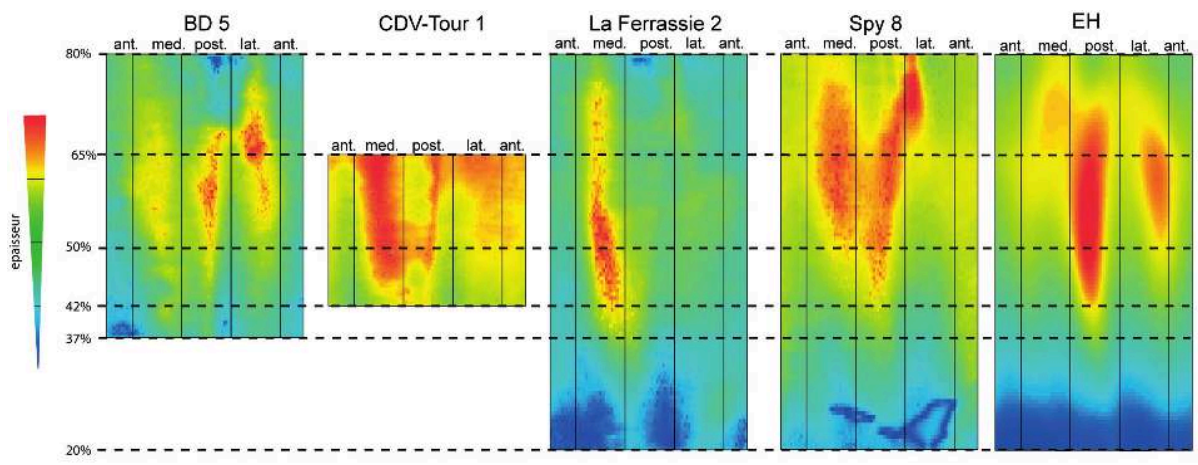

\section{Conclusions}

21 L'analyse comparative de l'organisation structurale externe et interne des diaphyses fémorales néandertaliennes BD 5 et CDV-Tour 1, issus du site de La Chaise-de-Vouthon, en Charente, mais séparées d'environ 80000 ans, a mis en évidence plusieurs caractéristiques communes entre ces deux spécimens adultes. Notamment, malgré des différences dimensionnelles vraisemblablement exprimant du dimorphisme sexuel, les deux fossiles partagent un contour périostéal subcirculaire au niveau de la midiaphyse, un contrefort médial (cf. Mussini et al. 2012), une linea aspera relativement mince associée à l'absence de pilastre. En effet, comme le montrent les résultats de l'analyse des propriétés de géométrie de section, dans les deux cas la partie proximale de la diaphyse est structurellement bien renforcée. De plus, la comparaison des cartographies morphométriques générées à partir du déroulement virtuel des spécimens indique la présence commune d'un épaississement médial du tissu cortical. Ainsi, comme d'autres fémurs néandertaliens, les diaphyses fémorales de La Chaise présentent une plus grande résistance aux contraintes de flexion orientées médiolatéralement, ce qui suggère une position plus latérale du corps au-dessus du support vertical (Trinkaus 1976, 2007), ainsi que l'influence sur la distribution et dissipation des charges biomécaniques de la morphologie pelvienne et des proportions des membres (Ruff 1995; Weaver et al. 2003 ; Trinkaus 2011 ; Trinkaus et Ruff 2012). Malgré des différences importantes dans les dimensions externes de la diaphyse fémorale (p.ex. Trinkaus 1980), la répartition topographique du tissu cortical révélée par les cartographies morphométriques ne met pas en évidence de spécificités liées au dimorphisme sexuel chez les fossiles de La Chaise. Enfin, par rapport à l'échantillon spécifiquement considéré dans cette étude et des paramètres quantitatifs mesurés, au cours de la période chronologique MIS 5-3 on ne remarque pas une tendance évolutive indiquant des changements évidents dans l'agencement endostructural et dans les propriétés biomécaniques de la diaphyse fémorale néandertalienne. Toutefois, il est important de noter ici la faible taille de l'échantillon du MIS 4 disponible à ce jour et, surtout, la nécessité dans les analyses futures de détailler les variations de l'os cortical 
sans discontinuité le long de la diaphyse (chaque $1 \%$ ) en utilisant un plus grand nombre de registres numériques à haute résolution.

\section{BIBLIOGRAPHIE}

ARMAND D. 1998 - La faune de la grotte Bourgeois-Delaunay, commune de La Chaise-de-Vouthon (Charente). Résultats préliminaires. Paleo, 10, p. 77-86.

BADDELEY A. 2008 - Analyzing spatial point patterns in R. Workshop notes. CSIRO Online Technical Publication www.csiro.au/resources/pf16h.html

BAYLE P., BONDIOLI L., MACCHIARELLI R., MAZURIER A., PUYMERAIL L., VOLPATO V. et ZANOLLI C. 2011 - Three-dimensional imaging and quantitative characterization of human fossil remains. Examples from the NESPOS database. In : Macchiarelli R. et Weniger G.-C. (eds.) Pleistocene Databases. Acquisition, Storing, Sharing. Wissenschaftliche Schriften des Neanderthal Museums 4, Mettmann, p. 29-46.

BEAUVAL C., MAUREILLE B., LACRAMPE-CUYAUBÈRE F., SERRE D., PERESSINOTTO D., BORDES J.G., COCHARD D., COUCHOUD I., DUBRASQUET D., LAROULANDIE V., LENOBLE A., MALLYE J.-B., PASTY S., PRIMAULT J., ROLLAND N., PÄÄBO S. et TRINKAUS E. 2005 - A late Neandertal femur from Les Rochers-de-Villeuneuve, France. Proceedings of the National Academy of Sciences, 102, p. 7085-7090.

BERTRAN P., CANER L., LANGOHR R., LEMEE L. et D’ERRICO F. 2008 - Continental palaeoenvironments during MIS 2 and 3 in southwestern France : the La Ferrassie rockshelter record. Quaternary Science Reviews, 27, p. 2048-2063.

BLACKWELL B.A., SCHWARCZ H.P. et DEBÉNATH A. 1983 - Absolute dating of hominids and Paleolithic artefacts of the cave of La Chaise-de-Vouthon (Charente), France. Journal of Archaeological Science,10, p. 493-513.

BLACKWELL B.A., PORAT N., SCHWARCZ H.P. et DEBÉNATH A. 1992 - ESR dating of tooth enamel : comparison with $230 \mathrm{Th} / 234 \mathrm{U}$ speleothem dates at La Chaise-de-Vouthon (Charente), France. Quaternary Science Reviews, 11, p. 231-244.

BLACKWELL B.A., BISSON M.S. ; SKINNER A.R. et BEELITZ P. 2007 - ESR dating bovid teeth from the Neandertal layer at La Ferrassie, France. GSA Denver Annual Meeting 28-31 October 2007.

BONDIOLI L., BAYLE P., DEAN C., MAZURIER A., PUYMERAIL L., RUFF C., STOCK J.T., VOLPATO V., ZANOLLI C. et MACCHIARELLI R. 2010 - Technical note : Morphometric maps of long bone shafts and dental roots for imaging topographic thickness variation. American Journal of Physical Anthropology, 142, p. 328-334.

CHURCHILL S.E. 1998 - Cold adaptation, heterochrony, and Neandertals. Evolutionary Anthropology, 7, p. 46-61.

COLEMAN M.N. et COLBERT M.W. 2007 - Technical note : CT thresholding protocols for taking measurements on three-dimensional models. American Journal of Physical Anthropology, 133, p. $723-725$. 
CONDEMI S. 2001 - Les néandertaliens de La Chaise. Paris : CTHS (Documents préhistoire 15), $178 \mathrm{p}$.

COUCHOUD I. 2006 - Étude Pétrographique et Isotopique de Spéléothèmes du Sud-Ouest de la France Formés en Contexte Archéologique : contribution à la Connaissance des Paléoclimats Régionaux du Stade Isotopique 5. Thèse de doctorat. Université Bordeaux-1, Bordeaux, $347 \mathrm{p}$.

DALEN L., ORLANDO L., SHAPIRO B., BRANDSTROM-DURLING M., QUAM R., GILBERT M., THOMAS P., DÍEZ FERNÁNDEZ-LOMANA J., WILLERSLEV E., ARSUAGA J.L. et GÖTHERSTRÖM A. 2012 Partial genetic turnover in neandertals : continuity in the East and population replacement in the West. Molecular Biology and Evolution, 29, p. 1893-1897.

DEBÉNATH A. 1974 - Position statigraphique des restes humains antewurmiens de Charente. Bulletins et Mémoires de la Société d'Anthropologie de Paris, 13, p. 417-426.

DEBÉNATH A. 1977 - The latest finds of antewürmien human remains in Charente (France). Journal of Human Evolution, 6, p. 297-302.

DEBÉNATH A. 2006 - Néanderthaliens et Cro-Magnons. Les Temps Glaciaires dans le Bassin de la Charente. Paris : Le Croît Vif, 356 p.

DEGIOANNI A., FABRE V. et CONDEMI S. 2011 - Génétique et paléoanthropologie : deux approches pour un dialogue autour des Néandertaliens. Bulletins et Mémoires de la Société d'Anthropologie de Paris, 23, p. 1-18.

DI VINCENZO F., CHURCHILL S.E. et MANZI G. 2012 - The Vindija Neanderthal scapular glenoid fossa : Comparative shape analysis suggests evo-devo changes among Neanderthals. Journal of Human Evolution, 62, p. 274-285.

FABRE V. CONDEMI S. et DEGIOANNI A. 2009 - Genetic evidence of geographical groups among Neanderthals. Plos One, 44, e5151.

FAJARDO R.J., RYAN T.M. KAPPELMAN J. 2002 - Assessing the accuracy of high-resolution X-ray computed tomography of primate trabecular bone by comparisons with histological sections. American Journal of Physical Anthropology, 118, p. 1-10.

HEIM J.-L. 1976 - Les Hommes fossiles de la Ferrassie. I. Le gisement. Les squelettes adultes (crâne et squelette du tronc). Paris : Masson, p. 330.

HEIM J.-L. 1982 - Les Hommes fossiles de la Ferrassie. II. Les squelettes adultes (squelette des membres). Paris : Masson, p. 276.

HODGSON J.A., BERGEY C.M. et DISOTELL T.R. 2010 - Neandertal genome : The ins and outs of African genetic diversity. Current Biology, 20, p. 517-519.

JAUBERT J., MAUREILLE B. et TURQ A. 2010 - A stratigraphic and chronological revision of Neanderthal burials in Western Europe : Chronicle of a long-awaited aging. Paleoanthropology, 2010, p. 20.

MACCHIARELLI R., BONDIOLI L., DEBÉNATH A., MAZURIER A., TOURNEPICHE J.-F., BIRCH W. et DEAN C. 2006 - How Neanderthal molar teeth grew. Nature, 444, p. 748-751.

MARIOTTI V. et BELCASTRO M. 2011 - Lower limb entheseal morphology in the Neandertal Krapina population (Croatia, 130000 BP). Journal of Human Evolution, 60, p. 694-702.

MARTIN R. et SALLER K. 1956-1962 - Lehrbuch der Anthropologie. Stuttgart : Gustav Fischer Verlag, 3, p. 2999. 
MAZURIER A., NAKATSUKASA M. et MACCHIARELLI R. 2010 - The inner structural variation of the primate tibial plateau characterized by high-resolution microtomography. Implications for the reconstruction of fossil locomotor behaviours. Palevol, 9, p. 349-359.

MEYER V., BRUZEK J., COUTURE C., MADELAINE S., et MAUREILLE B. 2011 - Un nouveau bassin néandertalien. Paleo, 22, p. 207-222.

MUSSINI C., CREVECOEUR I., GARRALDA M.-D., MANN A. et MAUREILLE B. 2012 - A new Neandertal femoral diaphysis from Les Pradelles (Marillac-le-Franc, Charente, France). Periodicum Biologorum, 114, p. 117-123.

PEBESMA EJ. 2004 - Multivariable geostatistics in S : the gstat package. Computers and Geosciences, 30, p. 683-691.

PONCE DE LEON M.S., GOLOVANOVA L., DORONICHEV V., AKAZAWA T., KONDO O., ISHIDA H. et ZOLLIKOFER C.P.E. 2008 - Neanderthal brain size at birth provides insights into the evolution of human life history. Proceedings of the National Academy of Sciences, 105, p. 13764-13768.

PUYMERAIL L. 2011 - Caractérisation de l'endostructure et des propriétés biomécaniques de la diaphyse fémorale : la signature de la bipédie et la reconstruction des paléo-répertoires posturaux et locomoteurs des Hominines. Thèse de Doctorat. Muséum national d'Histoire naturelle, Paris, $513 \mathrm{p}$.

PUYMERAIL L., VOLPATO V., DEBÉNATH A., MAZURIER A., TOURNEPICHE J.-F. et MACCHIARELLI R. 2012a - A Neanderthal partial femoral diaphysis from the "grotte de la Tour", La Chaise-deVouthon (Charente, France) : Outer morphology and endostructural organization. Palevol, 11, p. 581-593.

PUYMERAIL L., RUFF C.B., BONDIOLI L., WIDIANTO H., TRINKAUS E. et MACCHIARELLI R. 2012b Structural analysis of the Kresna 11 Homo erectus femoral shaft (Sangiran, Java). Journal of Human Evolution, 63, p. 741-749.

PUYMERAIL L., MARCHAL F., CHAUMOITRE K., PANUEL M. et MACCHIARELLI R. 2012c Architecture de la diaphyse fémorale et évaluation quantitative de l'influence de la masse musculaire et de la conformation pelvienne chez Homo sapiens. Bulletins et Mémoires de la Société d'Anthropologie de Paris, 25 p. S35-S36.

RASBAND W.S. 2010 - ImageJ. U. S. National Institutes of Health, Bethesda, Maryland, USA. http://rsb.info.nih.gov/ij/

RUFF C.B. 1995 - Biomechanics of the hip and birth in early Homo. American Journal of Physical Anthropology, 98, p. 527-574.

RUFF C.B. 2008 - Biomechanical analyses of archaeological human skeletal samples. In : Katzenberg M.A. et Saunders S.R. (eds.) Biological Anthropology of the Human Skeleton, second ed. Wiley-Liss, Hoboken, p. 183-206.

RUFF C.B., MCHENRY H.M. et THRACKERAY J.F. 1999 - Cross-sectional morphology of the SK 82and 97 proximal femora. American Journal of Physical Anthropology 109 :509-521

SCHWARCZ H.P. et DEBENATH A. 1979 - Datation absolue des restes humains de La Chaise-deVouthon (Charente) au moyen du déséquilibre des séries d'Uranium. C.R. Academie des Science Paris, Ser II, 288, p. 1155-1157.

SEMAL P., ROUGIER H., CREVECOEUR I., JUNGELS C., FLAS D., HAUZEUR A., MAUREILLE B., GERMONPRE M., BOCHERENS H., PIRSON S., CAMMAERT L., DE CLERCK N., HAMBUCKEN A., HIGHAM T., TOUSSAINT M. et VAN DER PLICHT J. 2009 - New data on the late Neandertals : direct dating of the Belgian Spy fossils. American Journal of Physical Anthropology, 138, p. 421-428. 
SLÁDEK V., BERNER M., GALETA P., FRIEDL L. et KUDRNOVA S. 2010 - Technical note : The effect of midshaft location on the error ranges of femoral and tibial cross-sectional parameters. American Journal of Physical Anthropology, 141, p. 325-332.

SPOOR F., ZONNEVELD F. et MACHO G.A. 1993 - Linear measurements of cortical bone and dental enamel by computed tomography : applications and problems. American Journal of Physical Anthropology, 91, p. 469-484.

STEWART J.R. et STRINGER C.B. 2012 - Human evolution Out of Africa : The role of refugia and climate change. Science, 335, p. 1317-1321.

TRINKAUS E. 1976 - The evolution of the hominid femoral diaphysis during the Upper Pleistocene in Europe and the Near East. Zeitschrift fur Morphologie und Anthropologie, 67, p. 291-319.

TRINKAUS E. 1980 - Sexual differences in Neanderthal limb bones. Journal of Human Evolution, 9, p. 377-397.

TRINKAUS E. 2007 - Activité, stress et survie chez les Néandertaliens. In : Vandermeersch B. et Maureille B. (eds.) - Les Néandertaliens. Biologie et Cultures. Paris : CTHS, p. 131-137.

TRINKAUS E. 2011 - The postcranial dimensions of the La Chapelle-aux-Saints 1 Neandertal. American Journal of Physical Anthropology, 145, p. 461-468.

TRINKAUS E. et RUFF C.B. 1989 - Diaphyseal cross-sectional geometry and biomechanics of the Fond-de-Forêt 1 femur and the Spy 2 femur and tibia. Anthropologie et Préhistoire, 100, p. 33-42.

TRINKAUS E. et RUFF C.B. 1999 - Diaphyseal cross-sectional geometry of Near Eastern Middle Palaeolithic humans : The femur. Journal of Archeological Science, 26, p. 409-424.

TRINKAUS E. et RUFF C.B. 2012 - Femoral and tibial diaphyseal cross-sectional geometry in Pleistocene Homo. PaleoAnthropology, 2012, p. 13-62.

TRINKAUS E., RUFF C.B., CHURCHILL S.E. et VANDERMEERCH B. 1998 - Locomotion and body proportions of the Saint-Cèsaire 1 Châtelperronian Neandertal. Proceedings of the National Academy of Sciences, 95, p. 5836-5840.

VIEILLEVIGNE E., BOURGUIGNON L., ORTEGA I. et GUIBERT P. 2008 - Analyse croisée des données chronologiques et des industries lithiques dans le grand sud-ouest de la France (OIS 10 à 3 ). Paleo, 20 , p. $145-166$.

VOLPATO V., COUTURE C., MACCHIARELLI R., VANDERMEERSCH B. 2011 - Endostructural characterization of the Regourdou 1 Neanderthal proximal arm : bilateral asymmetry and handedness. In : CONDEMI S. et WENIGER G.C. (eds.), Continuity and Discontinuity in the Peopling of Europe. Vertebrate Paleobiology and Paleoanthropology Series. Springer, New York, p 175178.

VOLPATO V., FRAYER D.W., MACCHIARELLI R., GUATELLI-STEINBERG D., FIORE I. et BONDIOLI L. 2012 - Hand to mouth in a Neandertal : right handedness in Regourdou 1. Plos One, 7, e43949.

VOLPATO V., MAZURIER A., PUYMERAIL L. et MACCHIARELLI R. 2013 - Lower limb. Spy I and II. Internal structure of the femurs and tibia. In : SEMAL P. et TOUSSAINT M. (eds.), Spy Cave. State of 120 Years of Pluridisciplinary Research on the Betche-aux-Rotches from Spy (Jemeppe-surSambre, Province of Namur, Belgium). Bruxelles : Institut Royal des Sciences Naturelles de Belgique, (sous presse).

WALKER M.J., ORTEGA J., PARMOVÁ K., LÓPEZ M.V. et TRINKAUS E. 2011 - Morphology, body proportions, and postcranial hypertrophy of a female Neandertal from the Sima de las Palomas, southeastern Spain. Proceedings of the National Academy of Sciences, 108, p. 10087-10091. 
WEAVER T.D. 2003 - The shape of the Neandertal femur is primarily the consequence of a hyperpolar body form. Proceedings of the National Academy of Sciences, 100, p. 6926-6929.

WEAVER T.D. 2009 - The meaning of Neandertal skeletal morphology. Proceedings of the National Academy of Sciences, 106, p. 16028-16033.

WEAVER T.D. et HUBLIN J.J. 2009 - Neandertal birth canal shape and the evolution of human childbirth. Proceedings of the National Academy of Sciences, 106, p. 8151-8156.

WOOD S.N. 2006 - Generalized additive models : an introduction with R. Boca Raton : Chapman \& Hall, $410 \mathrm{p}$.

\section{RÉSUMÉS}

Les diaphyses fémorales néandertaliennes BD 5 (MIS 5e), représentant un jeune adulte de sexe féminin, et CDV-Tour 1 (MIS 3), un sujet adulte de sexe masculin, proviennent tous les deux du site de La Chaise-de-Vouthon, en Charente, mais sont séparées d'environ 80000 ans. Dans le but de mettre en évidence d'éventuels changements évolutifs de nature morpho-structurelle au cours de ce laps de temps, nous présentons ici l'analyse comparative de leur structure externe et interne. En plus des caractéristiques morphologiques et des variations des diamètres externes au niveau mi-diaphysaire et de la section sous-trochantérienne, nous avons quantifié, à partir de leur registre microtomographique $(\mu \mathrm{CT})$, les propriétés géométriques de sections de ces spécimens à $50 \%, 65 \%$ et $80 \%$ de la longueur biomécanique et réalisé la cartographie morphométrique de la distribution topographique du tissu cortical. Malgré des différences vraisemblablement liées au dimorphisme sexuel, les deux diaphyses de La Chaise partagent, entre elles, mais aussi avec l'ensemble des fémurs néandertaliens européens considérés dans cette étude, les caractéristiques structurales typiques de ce taxon. En effet, au moins par rapport aux paramètres quantitatifs mesurés et aux spécimens disponibles à ce jour pour ce type d'analyses, au cours de la période chronologique MIS 5-3 nous n'avons pas remarqué de changements évidents dans l'agencement endostructural et dans les propriétés biomécaniques de la diaphyse fémorale néandertalienne.

Neanderthal femoral shaft of BD 5 (MIS 5e), representing a young adult female, and CDV-Tour 1 (MIS 3), an adult male, are both from the site of La Chaise-de-Vouthon, Charente, but are separated by about 80,000 years. In order to identify possible evolutionary morpho-structural changes during this time span, we present a comparative analysis of their internal and external structure. In addition to the morphological characteristics and changes in their external diameters at midshaft and subtrochanteric levels, we quantified, from their microtomographic record $(\mu \mathrm{CT})$, the cross-sectional geometric properties of these specimens at $50 \%, 65 \%$ and $80 \%$ of the biomechanical femoral length and analyzed the morphometric maps of the topographical distribution of cortical bone. Despite differences likely related to sexual dimorphism, both shaft of La Chaise share among themselves but also with all European Neanderthals femurs considered in this study, the typical structural features of this taxon. Indeed, at least compared to the quantitative parameters measured and specimens available for this type of analysis, during the time period MIS 5-3 we did not notice any obvious changes in the endostructural arrangement and in the biomechanical properties of the Neanderthal femoral shaft. 
INDEX

Mots-clés : La Chaise-de-Vouthon, fémurs néandertaliens, endostructure, biomécanique

Keywords : La Chaise-de-Vouthon, Neanderthal femurs, endostructure, biomechanics

\section{AUTEURS}

\section{LAURENT PUYMERAIL}

Unité d'Anthropologie bio-culturelle, Droit, Éthique \& Santé (ADÉS), UMR 7268, Université d'AixMarseille-EFS-CNRS, FR - 13344 Marseille - laurent.puymerail@univ-amu.fr

\section{SILVANA CONDEMI}

Unité d'Anthropologie bio-culturelle, Droit, Éthique \& Santé (ADÉS), UMR 7268, Université d'AixMarseille-EFS-CNRS, FR - 13344 Marseille - silvana.condemi@univ-amu.fr

\section{ANDRÉ DEBÉNATH}

Département de Préhistoire, UMR 7194, Muséum national d'Histoire naturelle FR-75005 Paris andre.debenath@gmail.com 